\title{
The influence of an improved hydrodynamic model on breakwaters design
}

\author{
H. Diab ${ }^{1,2}$, P. Lafon ${ }^{1} \&$ R. Younes ${ }^{2}$ \\ ${ }^{1} I C D$, LASMIS, Université de Technologie de Troyes, France \\ ${ }^{2}$ Lebanese University, Lebanon
}

\begin{abstract}
The influence of hydrodynamic model selection on the optimal layout of fixed breakwaters found in harbour design has been tested. Results from different models were compared. The mild slope equation was considered as reference. An extended mild slope equation taking into account the slope and curvature of rapid variation in the bathymetry was examined. The influence of adding the wave dissipation factor like bottom friction and wave breaking phenomena were verified. The effect of taking into account the irregularity of incident wave was examined comparing to monochromatic waves. This study was built on testing a genetic algorithm optimization process to identify the optimal shape and location of fixed breakwaters where the degree of attenuation of waves in the harbour zone was defined as object. The hydrodynamic model was varied and the results were compared. Three case studies with different bathymetries and boundary conditions were taken. The case of deep harbour where wave breaking didn't have a great influence was the first. Then a case of shallow harbour is discussed and finally an open sea case was studied as typical big gas ship refuges in a random wave area.
\end{abstract}

Keywords: breakwater, hydrodynamic modelling, sea wave, harbour.

\section{Introduction}

Harbours have always remained the central nodes of world trade. Designing harbours in the adequate way to maintain its safety and functionability is essential for coastal engineers and researchers. Building defence structures as breakwaters has the role of attenuating waves inside the harbour. The layout of breakwaters has to be a result of wave-structure interaction problem. The hydrodynamic model forms the main parameter of the problem. Wave 
attenuation will be the objective, and wave forces acting on the structure will be one of the principle constraints in any coastal project. Too many authors worked on putting and improving the wave modelling theories to simulate the distribution of wave height, its dynamic pressure and velocity different components. In view of the excessive computational efforts required to solve a sophisticated hydrodynamic model, and the great number of possibility to be tested before choosing an optimum design of breakwater with the minimum possible cost, in a domain where the investment cost are big. To minimize the computational cost, authors worked on many approach, they used optimisation techniques that allowed finding the optimum solution in the least computational time, and they choose often to abandon a lot of phenomena that occur in oceans and used a light hydrodynamic model.

In this paper we will test the influence of the hydrodynamic model selection on the optimal layout of a fixed breakwater. To do this we will refer to an optimisation study of fixed breakwater based on genetic algorithm used the basic mild slope equation known as Berkhoff equation to solve the hydrodynamic constraints (Berkhoff [1]).

In section 2, the different levels of the hydrodynamic model are presented. The basic mild slop equation is explained. The extended mild slop equation which could be applied in a rapid varying bathymetry is added. The dissipation phenomena due to bottom friction and wave breaking are taken into account. Then the model is generalized to have an irregular wave incident instead of monochromatic wave.

In section 3, the optimisation problem is defined then optimisation approach used in Elchahal et al. [2] is summarised. The tests are explained in section 4, where the domains of studies are defined and the way of comparison is developed. Results are shown and analysed in section 5 and finally a general conclusion is derived in section 6 .

\section{Hydrodynamic modelling}

The mild slop equation eqn. (1) remains as one of the main models in coastal engineering problems to calculate the wave field. It was derived by Juri Berkhoff in 1972. It can approximate the velocity potential in steady state at any point $(x, y)$ of a varying bathymetry region, taking into account both refraction and diffraction. It is called by mild slope equation because it was only valid for mild slop bathymetries Berkhoff [1].

$$
\text { จ. }\left(C C_{g} \nabla \emptyset\right)+C C_{g} k^{2} \emptyset=0
$$

$C$ and $C_{g}$ are, respectively, the phase velocity and the group velocity, $\mathrm{k}$ is the wave number and $\varnothing$ is the velocity potential in xy plane.

This partial differential equation is easy to be solved numerically. By solving the frequency dispersion relation eqn. (2), $k$ could be calculated in function of bottom depth $h$ Airy [3].

$$
\frac{w^{2}}{g k}=t h(k h)
$$

$w$ is the wave pulsation, $g$ is the gravity acceleration. 
And then $C$ and $C_{g}$ become determined.

$$
\begin{aligned}
& C=\frac{w}{k} \\
& C_{g}=\frac{1}{2}\left[1+\frac{2 k h}{\operatorname{sh}(2 k h)}\right] C
\end{aligned}
$$

The boundary conditions are Neumann boundary. A boundary could be an incident boundary eqn. (5), a radiation boundary eqn. (6) and a partial or total reflection boundary eqn. (7).

$$
\begin{aligned}
& \frac{\partial \emptyset}{\partial n}-i k \cos \theta_{p} \emptyset=\frac{\partial \gamma}{\partial n}-i k \cos \theta_{p} \gamma \\
& \frac{\partial \emptyset}{\partial n}-i k \cos \theta_{p} \emptyset=0 \\
& \frac{\partial \emptyset}{\partial n}-i \frac{1-R e^{i \propto}}{1+R e^{i \alpha}} k \cos \theta_{p} \emptyset=0
\end{aligned}
$$

$n$ is the normal vector to the boundary, $\theta_{p}$ is the angle between $\mathrm{n}$ and the wave normal, $\gamma$ is the imposed potential, $R$ is the reflection coefficient and $\propto$ is the time lag.

Noting that a radiation boundary is a reflection boundary has a reflection coefficient equal to zero.

Thereafter, many authors have introduced improvements on the main equation. Extended and modified mild slop equations appeared. In this section we will see some of these improvements.

\subsection{Extended mild slop equation}

The validity of the eqn. (1) was generalized for any type of bathymetry. The gradient and curvature terms was integrated to obtain an extended equation (eqn. (8))

$$
\begin{gathered}
\nabla \cdot\left(C C_{g} \nabla \emptyset\right)+(1+f) \cdot C C_{g} k^{2} \emptyset=0 \\
f=E_{1}(k h) \cdot(\nabla h)^{2}+\frac{E_{2}(k h)}{k_{0}} \cdot \Delta h
\end{gathered}
$$

$k_{0}$ is the wave number at an infinite depth.

Several expressions of $E_{1}$ and $E_{2}$ exist in the literature, we used the ones proposed in Chamberlain et al. [4].

with $x=2 \mathrm{kh}$.

$$
\begin{gathered}
E_{1}=\frac{\left\{x^{4}+4 x^{3} \sinh (x)-9 \sinh (\mathrm{x}) \sinh (2 \mathrm{x})+3 \mathrm{x}(\mathrm{x}+2 \sinh (\mathrm{x})) \cdot\left(\cosh ^{2}(\mathrm{x})-2 \cosh (\mathrm{x})+3\right)\right\}}{3\left(1+\frac{x}{\sinh (x)}\right) \cdot(x+\sinh (x))^{3}} \\
E_{2}=\frac{\{\sinh (x)-x \cdot \cosh (x)\}}{2\left(1+\frac{x}{\sinh (x)}\right) \cdot \cosh ^{2}(x / 2) \cdot(x+\sinh (x))}
\end{gathered}
$$

The first one represents the gradient term; it is multiplied by the square of the gradient. The second one relied to the Laplacian which represents the curvature.

\subsection{Energy dissipation}

In order to make the equation closer to the real physics, introducing the dissipation phenomena is necessary. Two kind of dissipative effects were taken into account; the bathymetric breaking and the bottom friction. A modification on the mild slope equation is suggested De Girolamo et al. [5]. 


$$
\nabla \cdot\left(C C_{g} \nabla \emptyset\right)+C C_{g}\left(k^{2} \cdot(1+f)+i k \mu\right) \emptyset=0
$$

$\mu$ is the dissipation coefficient.

\subsubsection{Bottom friction}

According to the nature of the bed, bottom friction could or couldn't have a great influence on the wave energy. That is why a friction coefficient must be added. Many formulation of dissipation coefficient to introduce bottom friction were suggested, some of them are simpler to compute than others (Putnam and Johson [6]).

$$
\mu=\frac{2 f_{w} H w^{3}}{3 \pi g C_{g} \sinh ^{3}(k h)}
$$

$f_{w}$ is the friction coefficient, $H$ is the wave height.

This coefficient depends directly from wave height which will be derived from the resultant velocity potential through the following relation:

$$
H=\frac{2 w}{g}|\varnothing|
$$

The equation is no more linear, introducing the dissipation makes it nonlinear which create more difficulties and computational effort requirements.

\subsubsection{Wave breaking}

Wave breaking is a different term of energy dissipation in wave propagation. Although it is a nonlinear term as the bottom friction, it is not as continuous as the friction. Wave breaking happens only when the velocity of a water particle at the free surface exceeds the wave propagation velocity. A critical breaking height $H_{m}$ must be calculated (Mei [7]).

$$
H_{m}=\frac{0.88}{k} \operatorname{th}\left(\frac{0.8}{0.88} \mathrm{kh}\right)
$$

The breaking occurs when $H$ exceeds $H_{m}$. One of the easiest ways to introduce it is to multiply the dissipation coefficient by a new term $Q_{b}$ as following Battjes et al. [8].

$f$ is the frequency.

$$
\mu=Q_{b} \frac{2 f H}{h C_{g}}
$$

$$
Q_{b}=\left\{\begin{array}{l}
0, H<H_{m} \\
1, H \geq H_{m}
\end{array}\right.
$$

\subsection{Random wave incident}

A wind-induced wave in the ocean will never be a monochromatic wave. It is a superposition of several monochromatic waves with different periods and lags.

Two approaches to model a random wave exist; the time domain approach and the spectral approach. Since that the solution of Berkhoff equation is based on the frequency dispersion relation, the second approach is used. To solve the equation with random wave input, an inverse direction is taken. The random wave is decomposed into specific number of representative monochromatic waves.

The incident wave is characterised by its proper significant height $H_{s}$ and peak period $T_{p}$, A spectral density is created. One of the most common is the 
JONSWAP spectrum (Hasselmann et al. [9]). Then a cumulative spectral density is derived. Then the curve is decomposed into equal energy parts, and the periods of monochromatic waves will be the mean period of each part. The wave heights will be the same for all the monochromatic waves.
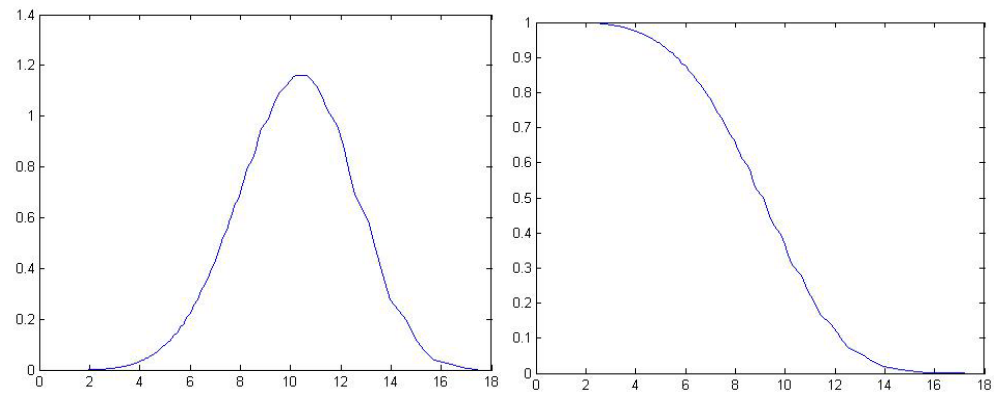

Figure 1: JONSWAP density spectrum (left) and cumulative spectrum (right).

Since the energy of each incident monochromatic wave is the same, the total energy at a point in the domain is the sum of the monochromatic wave energies making it up (Goda [10]).

$$
H=\sqrt{\frac{1}{N} \sum_{k}^{N} H_{k}^{2}}
$$

$N$ is the number of representative monochromatic waves.

Noting that in the case of random wave input, the results are in term of energy height, which is combined from the monochromatic waves' energies. That means we don't have any more complete information about velocity potential in the wave field, we only have the module of the potential eqn. (14). These mean we couldn't calculate the surface elevation in the field eqn. (19).

$$
\xi=\frac{w}{g} \operatorname{Real}(i \emptyset)
$$

$\xi$ is the surface elevation.

\section{Optimisation problem}

The optimisation problem is to find a design variable vector $\mathrm{x}_{\mathrm{i}}$, in manner to minimize the objective function $f\left(x_{i}\right)$ and subject to the constraint: $C\left(x_{i}\right) \leq 0$.

\subsection{Objective function}

The objective is to minimise the cost of the breakwater. We seek the optimal layout of a one segment breakwater that has a minimum length and a position with minimum depth to achieve an acceptable wave disturbance inside the target zone.

The objective function eqn. (20) is related to the length of breakwater and the depth in its position. The width of breakwaters is considered to be the same always. And the difference in the cost of volume unit according to the depth and distance from the coast is ignored. The cost is expressed in units of area. 
$f_{o b j}\left(x_{i}\right)=\operatorname{Min}\left[\left(\min \left(h_{2}, h_{1}\right)+\operatorname{abs}\left(\frac{h_{2}-h_{1}}{2}\right)\right) \cdot \sqrt{\left(x_{2}-x_{1}\right)^{2}+\left(y_{2}-y_{1}\right)^{2}}\right]$

$h_{1}$ and $h_{2}$ are respectively the depth of breakwater extremities $\left(x_{1}, y_{1}\right)$ and $\left(x_{2}, y_{2}\right)$.

\subsection{Constraints}

The only constraint has to be put in the problem is the wave disturbance constraint eqn. (21). A maximum allowable wave height $a$ in the target zone is considered.

$$
C\left(x_{i}\right)=\max (H(x, y, t)) \leq a
$$

Other constraints depend on the geometry of the domain and the navigational consideration must be taken into account properly in each case. They appear in the domain definition of the solutions. The solutions mustn't be outside a predefined polygon that respects these constraints.

\subsection{Genetic algorithm}

The optimisation approach proposed by Elchahal et al. [2] has been taken. The Genetic Algorithm (GA) is used. A Genetic Algorithm is a stochastic global search algorithm. It works in manner similar to the biological genetic. An initial population is generated randomly. Then a fitness equation takes the role to give each individual a degree of adaptation to the problem. Then the best solutions of the population are selected. After that a new generation is made through the crossover and mutation operators. The crossover operation is an interchange of genetic information between two selected individuals called parents to create a new individual. The mutation is the introducing of new random individuals in the generation, usually the rate of the mutation is low to grantee the convergence. This process is repeated sufficiently, until we got a well adapted generation to the fitness criteria.

\section{Test cases}

The aforementioned levels of the hydrodynamic model were substituted in the optimisation model. Three types of domains used to make the tests. The first one is a deep water harbour where the rapid change of bathymetry and the bottom friction were tested. A shallow port is used to test the breaking wave effect. An open see case is used finally to study the influence of random wave input.

In each case the domain with its proper bathymetry is defined. The target zone to be protected is chosen. And the polygon that contains the set of possible solutions is determined. A total of 7 tests were made, these different tests are shown in table 1.

\subsection{Deep harbour}

The incident wave comes from the NE side doing an angle of $225^{\circ}$ with the $\mathrm{x}$ axis. It has $3 \mathrm{~m}$ height and $9 \mathrm{~s}$ period. The west side is a radiation side open to the 
Table 1: $\quad$ Test cases.

\begin{tabular}{|l|c|c|c|}
\hline & Deep harbour & Shallow harbour & Open sea \\
\hline Basic mild slop & $\times$ & $\times$ & $\times$ \\
\hline Extended mild slop & $\times$ & & \\
\hline Bottom friction included & $\times$ & & \\
\hline Wave breaking included & & $\times$ & \\
\hline Random wave incident & & & $\times$ \\
\hline
\end{tabular}

sea. The reflexion boundary has a reflexion coefficient equal to 0.33 without any lag. The maximum allowable wave height in the target zone is $0.5 \mathrm{~m}$. The friction coefficient equal to 0.1 .

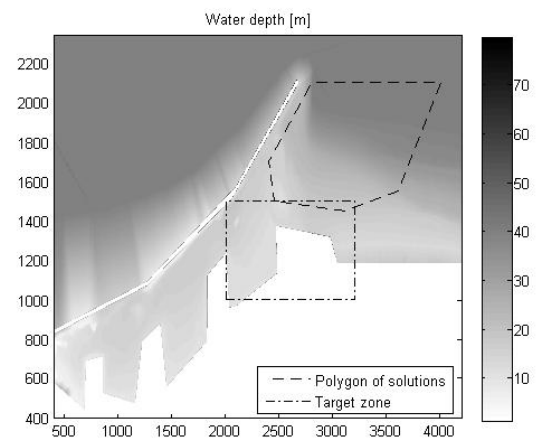

Figure 2: Deep harbour domain.

\subsection{Shallow harbour}

The incident wave comes from the SW side doing an angle of $45^{\circ}$ with the $\mathrm{x}$ axis. It has $2 \mathrm{~m}$ height and $4 \mathrm{~s}$ period. The east side is a radiation side open to the sea. The reflexion boundary has a reflexion coefficient equal to 0.33 without any
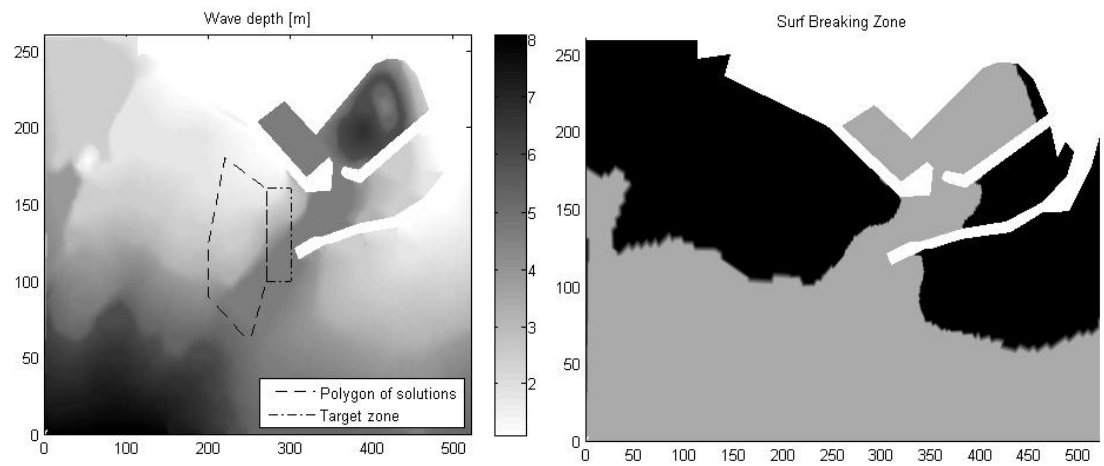

Figure 3: Shallow harbour domain. 
lag. The low depth of water near the shore makes the breaking phenomenon appears. The maximum allowable wave height in the target zone is $0.5 \mathrm{~m}$.

\subsection{Open sea case}

The incident wave comes from the NW side doing an angle of $315^{\circ}$ with the $\mathrm{x}$ axis. It has $2.8 \mathrm{~m}$ significant height and $10.4 \mathrm{~s}$ peak period. 5 representative waves are calculated using the method aforementioned in section 2.3; they are shown in table 2. The maximum allowable wave height in the target zone is $0.5 \mathrm{~m}$.

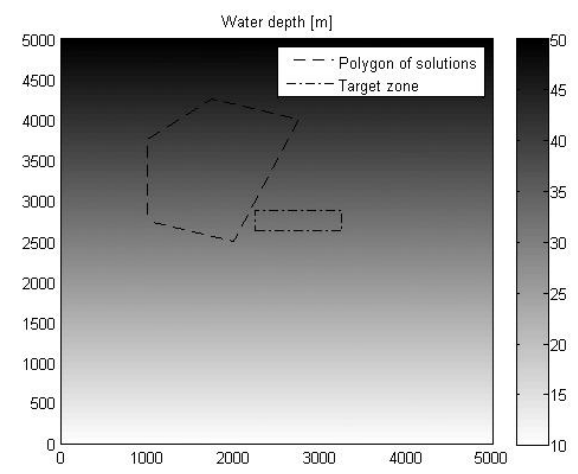

Figure 4: Open sea domain.

Table 2: Random wave calculated parameters.

\begin{tabular}{|l|l|l|l|l|l|}
\hline & Wave 1 & Wave 2 & Wave 3 & Wave 4 & Wave 5 \\
\hline$H(\mathrm{~m})$ & 0.85000 & 0.8552 & 0.8935 & 0.9281 & 0.8987 \\
\hline$T(\mathrm{~s})$ & 12.3200 & 10.6495 & 9.2400 & 7.6624 & 5.7120 \\
\hline
\end{tabular}

The east and south side are radiation sides open to the sea. The reflexion boundary has a reflexion coefficient equal to 0.33 without any lag. It exists only for the added breakwater.

This case simulates the offshore pumping station for the huge oil and gas tanker.

\section{Results and discussions}

The PDE Toolbox in MATLAB 2013 $\mathrm{a}^{\mathrm{TM}}$ is used to find the solution of the linear and nonlinear partial differential equation. The optimisation algorithm is coded with the reference functions of MATLAB ${ }^{\mathrm{TM}}$. All the testes have been run on the same computer to compare the consumed computational efforts by comparing the overall duration. A laptop with core i5-3210M CPU @ 2.50GHz and 6GB RAM is used.

The optimal solution of each case mentioned in section 4 is found and compared to reference solution of the same domain using the basic mild slop 
equation eqn. (1) to verify the existence of influence. The results are discussed to see if ignoring of such phenomena overestimates or underestimates the solution. To maintain the convergence to the optimal solution, we didn't stop before attending a steady state phase in the objective function.
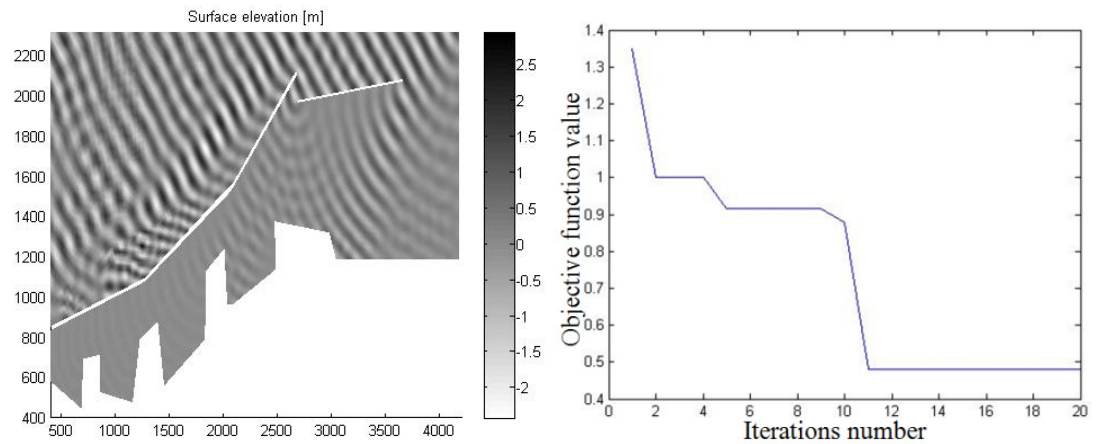

Figure 5: Optimal solution for reference case in deep harbour (left) and Objective function progress (right).

\subsection{Influence of rapid varying bathymetry}

The optimal layout has been determined after 20 iterations. Figure 6 shows the optimal solution achieved, after taking into consideration the rapid change bathymetry parameter. Results in table 3 show that the surface area of the breakwater is larger than it was in the reference case. That is logical, because ignoring the rapid change bathymetry in a domain with big slops and curvatures will disturb the wave field and usually underestimates the amount of wave energy, so it is obvious that the algorithm will tend to generate a bigger breakwater than the reference case.

They show also that adding the rapid change parameter has slightly affected the computational time. From this point it will be taken always into consideration.

\subsection{Influence of bottom friction}

Figure 6 shows the optimal solution achieved with taking bottom friction in consideration. In this case the area of the breakwater has been less than the reference case but the time cost has significantly increased, due to solve a nonlinear differential equation. Results, in table 3, show that ignoring bottom friction will overestimate the design.

Table 3: Deep harbour results.

\begin{tabular}{|l|l|l|l|}
\hline & Reference case & Rapid change & Bottom friction \\
\hline Computing time $(\mathrm{s})$ & 1119 & 1206 & 7100 \\
\hline Breakwater section $\left(\mathrm{m}^{2}\right)$ & 30000 & 38000 & 28000 \\
\hline
\end{tabular}



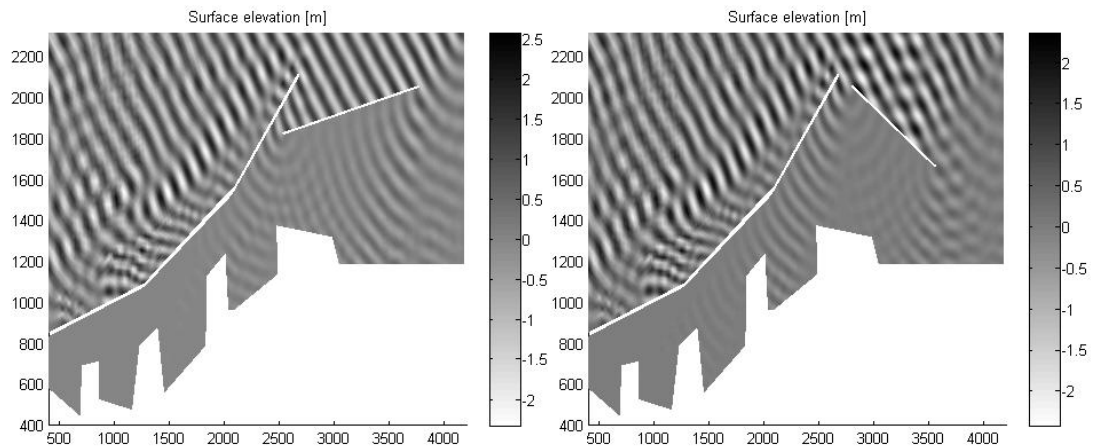

Figure 6: Optimal solution with rapid change parameter (left) and bottom friction (right).

\subsection{Influence of wave breaking}

In this case the optimal solution has shown a significant reduce of breakwater area, the wave energy has been dissipated due the wave breaking in surf zone shown in Figure 3. Results are summarized in table 4 and Figure 7 shows the optimal layout.

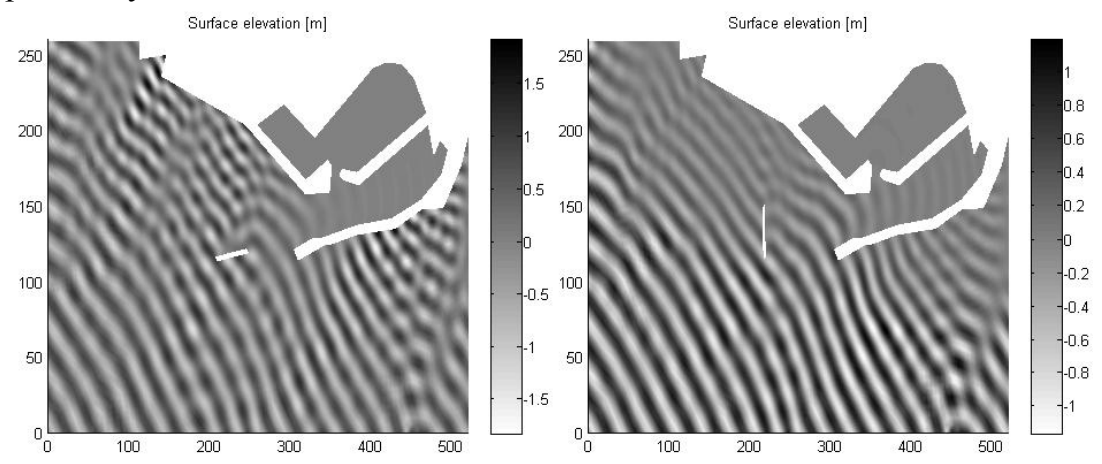

Figure 7: Optimal solution for reference case (left) and with wave breaking (right).

Table 4: Shallow harbour results.

\begin{tabular}{|l|l|l|}
\hline & Reference case & Wave breaking \\
\hline Computing time $(\mathrm{s})$ & 2290 & 16900 \\
\hline Breakwater section $\left(\mathrm{m}^{2}\right)$ & 120 & 80 \\
\hline
\end{tabular}

\subsection{Influence of random wave incident}

Random wave incident will affect hardly the computational time; it is $N$ times more than monochromatic wave, where $N$ is the number of representative waves. It has a great influence also on the breakwater section which had been reduced by $21 \%$. These results are shown in table 5 . The optimal solution is shown in 
Figure 8 in term of wave height because surface elevation couldn't be plotted using this method.

Table 5: Open sea results.

\begin{tabular}{|l|l|l|}
\hline & Reference case & Random wave \\
\hline Computing time $(\mathrm{s})$ & 13500 & 60600 \\
\hline Breakwater section $\left(\mathrm{m}^{2}\right)$ & 51000 & 40000 \\
\hline
\end{tabular}
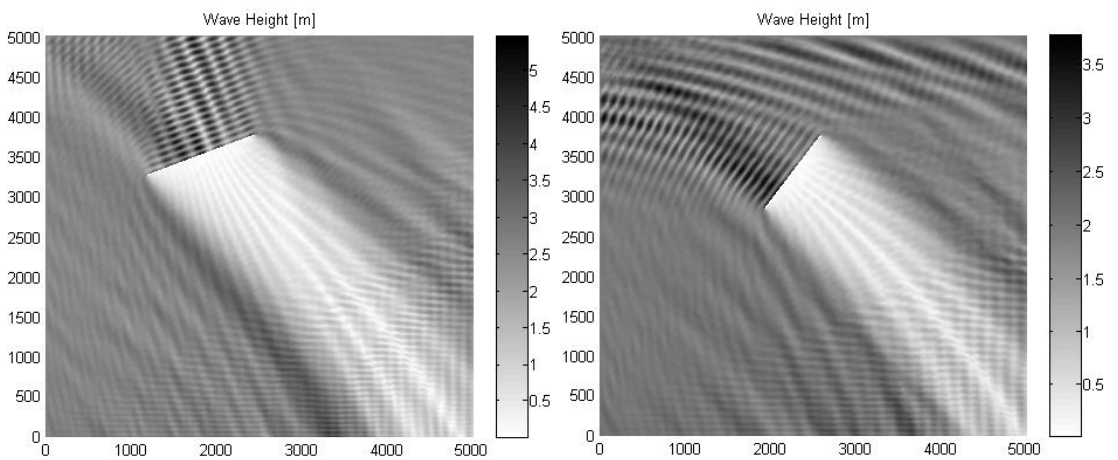

Figure 8: Optimal solution for reference case (left) and for random wave incident (right).

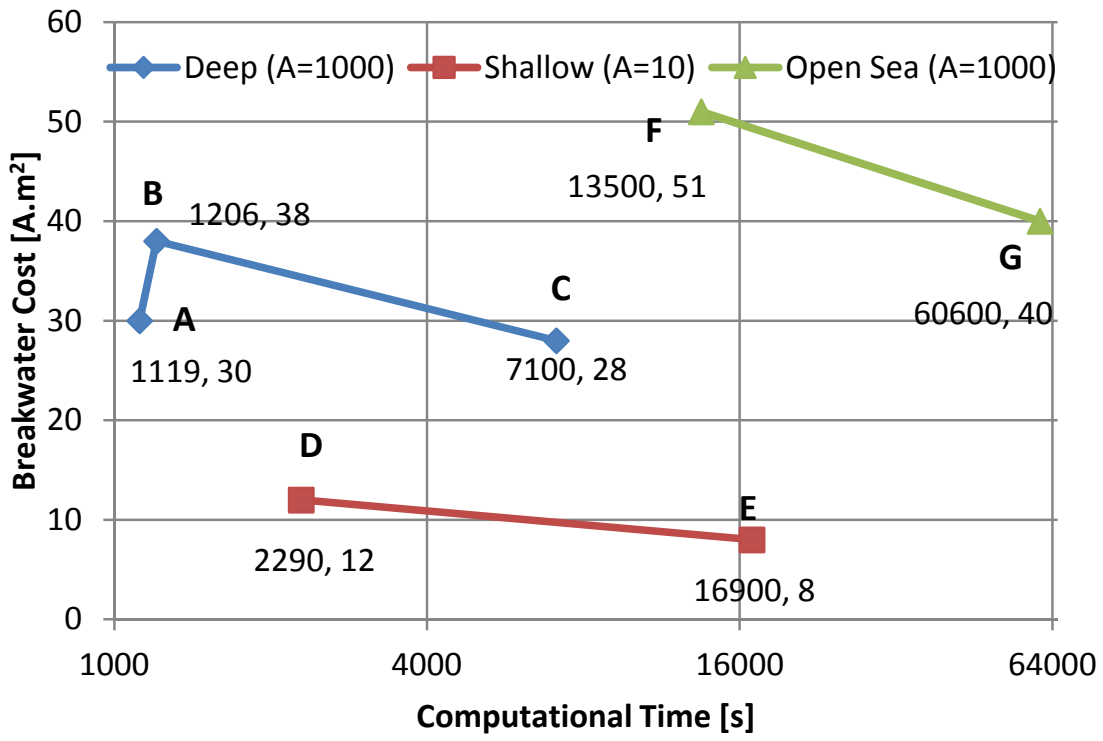

Figure 9: The cost of proposed breakwater in respect to the computational time consumed. A, D and $\mathrm{F}$ are the reference tests. $\mathrm{B}$ is the extended eqn. test, $\mathrm{C}$ is the bottom friction test, $\mathrm{E}$ is the wave breaking test and $\mathrm{G}$ is the random wave test. 
The differences show that it is very wrong to substitute a full analysis of a random wave by an approximation of monochromatic wave that has a wave height equal to $H_{s}$ and period equal to $T_{p}$.

\section{Conclusion}

Results in this paper showed the importance of taking the phenomena of real physics into consideration when designing coastal structure where the investment cost is always very high. Ignoring the rapid change bathymetry will underestimate the solution where ignoring the other phenomena will overestimate the solution. The energy dissipation depends a lot on the studied domain and specially the bathymetry. The random wave height couldn't be taken as a monochromatic wave where the results will become very different. The computational efforts limitation is a real challenge to implement all the phenomena together. Efficient optimisation approaches and appropriate numerical solutions of the hydrodynamic models need to be continuously sought.

\section{Acknowledgements}

This work was supported by the Regional Council of Champagne-Ardenne (RCCA) and the Lebanese University.

\section{References}

[1] Berkhoff, J.C.W., Computation of combined refraction-diffraction. Proc. of the 13th Coastal Engineering Conference, ASCE: Vancouver, pp. 471490, 1972.

[2] Elchahal, G., Younes, R. \& Lafon, P., Optimization of coastal structures: Application on detached breakwaters in ports. Ocean Engineering, 63, pp. 35-43, 2013.

[3] Airy, G., Tides and waves, Encyclopedia metropolitana (1817-1845). London, 1841.

[4] Chamberlain, P.G. \& Porter, D., The modified mild-slope equation. Journal of Fluid Mechanics, 291, pp. 393-407, 1995.

[5] De Girolamo, P., Kostense, J.K.. \& Dingemans, M.W., Inclusion of wave breaking in a mild-slope model. Proc. of the Int. Conf. On Computer Modelling in Ocean Engineering, Venise, 1988.

[6] Putnam, J.A. \& Johson, J.W., The dissipation of wave energy by bottom friction. Transactions, American Geophysical Union, 30(1), pp. 67, 1949.

[7] Mei, C.C., The Applied Dynamics of Ocean Surface Waves, World Scientific: 1989.

[8] Battjes, J.A. \& Janssen, J.P.F.M., Energy loss and set-up due to breaking random waves. Proc. of Coastal Engineering, ASCE: Hamburg, 1(16), 1978. 
[9] Hasselmann, K., Barnett, T.P., Bouws, E., Carlson, H., Cartwright, D.E. \& Enke, K. Measurements of wind-wave growth and swell decay during the Joint North Sea Wave Project (JONSWAP). Deutches Hydrographisches Institut: 1973.

[10] Goda, Y., Random Seas and Design of Maritime Structures. World Scientific: 2010 . 